\title{
The influence of coyote on Canada lynx populations assessed at two different spatial scales
}

\author{
A. Guillaumet 1,3 , J. Bowman², D. Thornton ${ }^{1}$ and D. L. Murray ${ }^{1}$ \\ ${ }^{1}$ Department of Biology, Trent University, DNA Building, Peterborough, ON K9J 7B8, Canada \\ ${ }^{2}$ Ontario Ministry of Natural Resources, Trent University DNA Building, Peterborough, Ontario K9J 7B8, Canada \\ ${ }^{3}$ Corresponding author.Email: albanguillaumet@gmail.com
}

Keywords: Canada lynx, Coyote, Distribution models, Fur harvest, Interspecific competition, Population trends, Spectral analysis, Time-series.

\begin{abstract}
Previous studies have suggested that the attenuation of Canada lynx (Lynx canadensis) cyclic dynamics with decreasing latitude may be the consequence of a reduced specialization on the lynx's primary prey, snowshoe hares (Lepus americanus). However, intraguild competitive interactions remain largely unexplored in situations where the temporal dynamics of food resources is pronounced, and lynx populations in the south of their distribution may be negatively affected by interspecific competition with other carnivores. In this paper, we used spectral analysis of fur harvest data collected at the state (US) and province (Canada) level to explore the spatial gradient of cyclic dynamics in lynx. Although some patterns were consistent with the 'diet specialization' hypothesis, we found that temporal variance of cycling propensity peaked at mid-latitudes, where transient, non-cyclic periods, coexisted with regular 10-year cycles. In these mid-latitude zones, non-cyclic periods did not coincide with loss of snowshoe hare cycling as demonstrated by historical records, and were not more frequent in recent decades as could be expected under a 'climatic forcing' scenario. Instead, we show that non-cyclic periods tended to coincide with periods of high coyote (Canis latrans) abundance and periods when coyotes apparently tracked snowshoe hare abundance as suggested by significant 10-year cycles lagging one or two years behind hare peaks. We used landscape-scale (trapline) fur harvest returns from five provinces in Canada to further probe the importance of interspecific competition in Canada lynx population dynamics. Accounting for coyote distribution and abundance did not bring additional explanatory and predictive power to models based solely on environmental and autecological predictors, suggesting that competition with coyote is not a force driving population abundance and cyclicity among lynx. We discuss the possible factors behind the apparent lack of consistency across spatial scales and recommend that further studies examine species interactions at a smaller (local) scale.
\end{abstract}

Abbreviations: GLM-Generalized Linear Model, IAD-N-inflation-adjusted + detrended harvest number, IA-N-inflation-adjusted harvest number, HPD-Highest Posterior Density, RLRT-Restricted Loglikelihood Ratio Test.

\section{Introduction}

The occurrence of interspecific competition between meso-carnivores is well known, with consequences of such interactions affecting species distribution, abundance and habitat use (Ritchie and Johnson 2009). For instance, the negative effect of coyote (Canis latrans Say) on various co-occurring fox species has been well documented, with interference competition (intra-guild predation) as well as exploitative competition (exploitation of similar prey) likely resulting in a general pattern of spatial segregation between coyote and fox species (e.g., Fedriani et al. 2000, Nelson et al. 2007). It follows that such interactions lead to local population decline or even suppression of fox species in the presence of the coyote (Kamler et al. 2003, Levi and Wilmers 2012).

The recognition that interspecific competition may mediate species decline has direct implications for the conservation of endangered species. The Canada lynx (Lynx canadensis Kerr; hereafter, lynx) was listed as a threatened species under the Endangered Species Act in the conterminous US states (US Fish and Wildlife 2000), with interspecific com- petition with other carnivores being regarded as a potential contributor to the recent demise of southern populations in southern Canada and Northern USA (Buskirk et al. 2000). The coyote is probably the most important competitor for lynx across the majority of its geographic range. The coyote is a more generalized feeder compared to lynx (e.g., Litvaitis and Harrison 1989, O'Donoghue et al. 1998), meaning that it may compete with lynx over the latter species' preferred prey (snowshoe hare, Lepus americanus Erxleben). For example, O'Donoghue et al. (1997) found that the numerical response of coyote and lynx to a snowshoe hare cycle in southwest Yukon were similar, and coyotes and lynx are the two most important mammalian predators of snowshoe hares throughout much of the boreal forest (O'Donoghue et al. 1998). Furthermore, coyotes do kill lynx, and there is speculation that coyotes could influence lynx more so than does the availability of snowshoe hares (Buskirk et al. 2000, Bayne et al. 2008). To date, however, direct evidence supporting the negative role of interference competition on lynx populations is lacking (Murray et al. 2008). 
The 9-11 year population cycle of the Canada lynx and its main prey, the snowshoe hare, is among the most well-known examples of cyclic population dynamics (Krebs et al. 2001). Strongly cyclic dynamics of the lynx in the boreal zone is attributed to its high degree of specialization on snowshoe hare ('diet specialization' hypothesis: Roth et al. 2007). Gradual loss of lynx cyclic dynamics towards the south end of its range is currently explained by the emergence of additional trophic interactions. First, this phenomenon is observed through apparent reduction in specialization of the lynx on snowshoe hare, attributed to the higher availability of alternative prey the lynx can switch to during periods of hare scarcity (Roth et al. 2007). Second, concurrently with loss of lynx cyclic dynamics, the apparent loss of truly cyclic snowshoe hare dynamics towards the south of the hare's range (Smith 1983) is potentially mediated by an increase in the number of generalist predators with which lynx may compete directly (Murray 2000 and references therein, Ripple et al. 2011). Hence, the attenuation of lynx cyclic dynamics could result, at least in part, from interspecific competition with other carnivores.

Despite its role in the development of community ecology theory and its long-recognized importance in explaining species distribution, abundance and 'regular' population fluctuations, intraguild competitive interactions remain largely unexplored in situations where the temporal dynamics of food resources is pronounced (Henden et al. 2010). In a review of the factors potentially explaining why some red-backed vole (Myodes gapperi (Vigors)) populations fluctuate with regular cycles rather than irregular fluctuations, Boonstra and Krebs (2012) considered interspecific competition as a plausible cause, as it could 'operate to keep populations perpetually low'. Ultimately they concluded that competition was not an important factor for red-backed voles, although its broader role among other taxa, including Canada lynx, remains to be determined.

In this paper, we first used a sliding time-window approach to explore spatio-temporal variability in the fur harvest records collected at the state (US) and province (Canada) level for lynx, compared to those for coyote (hereafter, our 'province-level' study). Our results reveal a degree of negative correlation between coyote numbers and the propensity for lynx cycles, perhaps suggesting that competition with coyotes may affect the cyclic dynamics of lynx, particularly in the core of the lynx range. We next investigated whether finer-scale harvest statistics for lynx and coyote also revealed evidence of competition between these carnivore populations (hereafter, our 'trapline-level' study). If lynx are negatively affected by interspecific competition with coyotes, then we expected that lynx population trends would be: i) skewed towards more positive values on traplines where the coyote was absent; and ii) negatively related to coyote population trends, when occurring in sympatry. Finally, we tested the hypothesis that patterns of coyote abundance should bring additional explanatory and predictive power to spatial models of lynx distribution and abundance based solely on environmental and autecological predictors (e.g., Guisan and Zimmermann 2000, Araujo and Luoto 2007).

\section{Materials and methods}

\section{I - Province-level study}

We used lynx and coyote fur harvest time series spanning 1919-2009 obtained from 12 Canadian provinces and territories and 4 US states (see Appendix 1 for data sources). We used centroid coordinates of each jurisdiction to spatially reference each time series. We used the uncorrected number of pelts in the harvest as our basic index of population size $(\mathrm{N})$. Several studies have shown a reasonable match between annual harvests and other types of population census (Cattadori et al. 2003, Ranta et al. 2008, Estay et al. 2011), suggesting that harvest data can be used as a proxy of population abundance especially for species having large numerical fluctuations through time. However, for harvest to generally provide a reliable index of population size, trapper effort and effectiveness should be constant or controlled for (Peacock and Garshelis 2006). Following Gosselink et al. (2003), harvest numbers were adjusted to their market price as a means of controlling for harvest effort (IA-N; Appendix 2). We also temporally detrended data by taking the residuals of a simple linear regression between $\mathrm{N}$ or IA-N and time (yielding D-N or IAD-N, respectively).

We used a spectral analysis based on Lomb-Scargle periodograms to test for the occurrence of population cycles (Glynn et al. 2006). Distinct peaks in the periodogram correspond to regular oscillations with distinct frequencies, which are inversely related to cyclic periods (Glynn et al. 2006). As the period of lynx cycles is about 10 years (e.g. Roth et al. 2007), we only estimated the periodograms for periods ranging from 5 to 15 years. Statistical significance of the highest peak (corresponding to the dominant harmonic) was used as a formal test of cyclicity (Glynn et al. 2006; we used $\alpha=0.05$ ). We also used the height of the highest peak, or spectral power, as a measure of the intensity of cyclicity, independently of whether it reached significance or not (Roth et al. 2007). The oscillatory period at peak was also computed and called "T_cycle". For each jurisdiction, we divided the 1919-2009 time-series into seven windows of thirty-one years, starting with the period 1919-1949, and then shifting to 1929-1959, and so on. The spectral analysis was conducted for each window, although we discarded those with a sample size $<20$. We tested for the presence of latitudinal gradients for parameters measuring population cyclicity (such as spectral power and T_cycle) using simple linear or quadratic regression. Spatial autocorrelation was accounted for by fitting a spatial simultaneous autoregressive lag model (lagsarlm in spdep package of R), where the weight of neighbors was modeled as an inverse function of distance: the weight of neighbor $j$ of focal jurisdiction $i, w[i, j]$, was given by $w[i, j]=1 /$ (spat. $\operatorname{dist}[i, j]+1)$.

In addition to the expected gradual decrease in average cyclic propensity with latitude, our sliding time-window approach revealed an unexpected peak in the temporal variability of cycling propensity at mid-latitudes (see Results). We tested two lines of evidence potentially supporting the influence of coyote competition in affecting lynx cyclic propensity 
at mid-latitudes, either through a direct or indirect dampening effect, as measured respectively by a negative relationship between lynx spectral power and coyote population size, or between lynx and coyote population sizes.

We first used an information-theoretic approach to test for a relationship between lynx spectral power and coyote population size. Lynx spectral power was our response variable, and we allowed different random intercepts for each midlatitude jurisdiction ( $\mathrm{n}=7$ Canadian provinces from British Columbia to Newfoundland) and time-window $(n=7)$ in a mixed-model setting, as Restricted Likelihood Ratio Tests (RLRT) based on 10,000 simulations (Scheipl et al. 2008) showed that the variance of these random effects was not null (both $\mathrm{P}<0.03$, not shown). Two main types of (fixed-effect) candidate factors were tested: i) the average lynx population size in the time-window (testing amplitude dampening; Ims et al. 2008); ii) the average coyote population size in the time-window, predicting a negative relationship if interspecific competition reduces cyclic propensity. We used AICc (Akaike's Information Criterion adjusted for small sample size) to evaluate models. Markov Chain Monte Carlo methods (function mcmcsamp in R 'Ime4' package) were used to estimate 95\% Highest Posterior Density (HPD) intervals for fixed-effect parameters (estimated from 10,000 samples from the posterior distribution).

We used three different methods to assess the significance of the synchrony between the time series of lynx and coyote harvest, while accounting for the serial correlation in the null. Analyses were performed at time-lag $\mathrm{t}=0$ and $\mathrm{t}=-1$, the latter by comparing the lynx time series from year 2 to 91 (19202009) with the coyote time series from year 1 to 90 (19192008). For block approaches at $t=0$, we present the results obtained when using lynx and coyote time series from year 1 to 90 to keep block size constant (ten blocks of size $b=9$, see below), but identical results were obtained when using the full length of time-series (from year 1 to 91). Analyses were conducted for two provinces that had sufficient data (Alberta and Saskatchewan; linear interpolation was used to estimate a single year of missing data in Saskatchewan).

We developed a block permutation approach using Spearman's rank correlation coefficient $\rho$ as our measure of interspecific synchrony (e.g., Buonaccorsi et al. 2001). While keeping the coyote time-series constant, we divided the lynx time-series into 10 non-overlapping blocks of length $\mathrm{b}=9$ years (the average period of cyclic fluctuation when they occurred, see Results and Roth et al. 2007). New lynx time-series were reconstructed by sampling without replacement from these 10 blocks, the objective being to generate time-series with correlated error terms that exhibited approximately the same pattern of dependence as the real errors (Davidson and MacKinnon, 2007). Ten thousand randomized lynx time series were constructed this way, Spearman's $\rho$ was computed for each to generate the distribution expected under H0 (no synchrony), and a one-tailed test was used to test for negative synchrony. We also performed: i) a moving block bootstrap as it may be more efficient than non-overlapping block approaches (Davidson and MacKinnon, 2007); and ii) a residual-based test described in Alpargu and Buonaccorsi
(2009), because conclusions may be sensitive to the choice of actual block length (Davidson and MacKinnon, 2007). These last two methods are detailed in Appendix 3.

Two possibly confounding factors, climate and hare dynamics, were also investigated as follows. First, we tested whether lynx cyclic dynamics tended to decrease through time, as could be expected under a 'climatic forcing' scenario (Brommer et al. 2010), by performing simple linear regression between time (as measured by the mid-year of each timewindow) and lynx spectral power, as well as simple linear regression between time and the estimated random intercepts for each time-window (i.e., the estimated contribution to lynx spectral power value due to each time-window while simultaneously accounting for other factors such as competition; see above). Long-term trends in North America suggest that time is a good proxy of climate change over the timeframe of our assessment (Karl et al. 2009, Zhang et al. 2010). Second, we used the graphical data presented in Smith (1983), based on the 17-year long Canadian Snowshoe Rabbit Enquiry, to identify hare cyclical peaks in nine Canadian provinces with significant hare cyclic patterns (from Yukon to Nova Scotia) over the course of the study period (1931-1947). Smith (1983) summarized the data as having either negative, null, or positive trends for each of the 370 grid cells (of 90 miles squared each) and each of the seventeen years. We identified hare cyclical peak for each province (except Nova Scotia) as the last year with predominantly positive trends that followed several years of increasing trends; two peaks were detected for each of the eight provinces, as could be expected from the national-wide average cyclic periodicity of 8.9 years (Smith 1983); peaks in Nova Scotia were inferred from the cycle phase (about 4 years ahead of Yukon) since graphical data in Smith (1983) were hard to interpret. We visually investigated whether peaks in hare fluctuations were followed by peaks in lynx and/or coyote fluctuations.

\section{II - Trapline-level study}

Data. Population harvest time series analysis was run independently for five different provinces of Canada (British Columbia, Alberta, Saskatchewan, Ontario and Quebec). Annual lynx and coyote fur harvest data were obtained from provincial wildlife management agencies; we limited our dataset to those provinces where harvest was subdivided into traplines or other spatial units that constituted our basic sampling unit. Province-wide data sets included all traplines, and we also built allopatry data sets including only traplines where lynx was present (at least one individual trapped) while coyote was absent, and sympatry data sets where both species were present. More information regarding the distribution of trapline size in each province (mean area and standard deviation of a single trapline) is given in Table 3.

We used the annual number of harvested pelts of Canada lynx and coyote per trapline as our basic index of population size, but combinations of the following supplemental 'control' runs were also performed (see Appendices 6-9 for details on which controls were performed for each analysis). First, pelt harvests were adjusted to their market price as a means of 
controlling for harvest effort (see above for details). Second, we corrected lynx abundance by red fox abundance as an additional control for harvest effort. Red fox is widespread and generally fairly common across the lynx and coyote ranges, which makes it suitable for that purpose. In that aim, we took the residuals of a simple linear regression between lynx and red fox abundance, or used red fox abundance as a covariate. Red fox harvests were adjusted to their market price as well in the controls where such a correction was applied to lynx and coyote. Following Weinstein (1977), we reasoned that trappers are 'opportunistic predators', and such generalist behavior can generate substantial positive covariation between meso-carnivore harvests (see also Discussion). Controlling for red fox abundance in harvests is an attempt to reduce such a bias. Finally, to account for a possible delay in lynx response, coyote abundance was also estimated at time-lag = -1 for spatial analyses.

For each trapline and year, we calculated a measure of spatial and temporal autocorrelation in lynx abundance (aut.s and aut.t covariates, respectively) as follows. For trapline i and year $j$, aut.s[i,j] was the average number of lynx in neighboring traplines $\mathrm{k}(\mathrm{k} \neq \mathrm{i})$, weighting each trapline as a function of its geographic distance (spat.dist, in kilometers) to the focal trapline $\mathrm{i}$, with weight $\mathrm{w}[\mathrm{i}, \mathrm{k}]=1 /$ (spat.dist $[\mathrm{i}, \mathrm{k}]+$ 1 ); aut.t[i,j] was the average number of lynx in trapline $i$ in previous and subsequent years of the time-series, weighting each year $1(1 \neq j)$ as a function of its temporal distance (spat. temp, in years) to the focal year $\mathrm{j}$ with weight $\mathrm{w}[\mathrm{j}, 1]=1 /$ spat. temp[j,1].

We used 19 bioclimatic variables (http://www.worldclim. org/bioclim) measuring aspects of temperature and precipitation. We obtained for each trapline a single value of each bioclimatic variable corresponding to the average over the period 1950-2000 and over all the pixels of the trapline. To reduce the number of predictors, the 19 bioclimatic variables were subjected to a Principal Component Analysis (PCA). The first four principal components (PC1 to PC4) were used for subsequent analyses as they explained a higher amount of variation than initial variables (i.e., their eigenvalue was $>1$ ).

Snow cover and snow depth were obtained from the North American Regional Reanalysis dataset (http://www.esrl.noaa. gov/psd/data/gridded/data.narr.htm), and used long-term winter means (Oct-Mar, averaged from 1979-2000). We also included the human influence index (see http://sedac.ciesin. columbia.edu/wildareas/methods.jsp for details). Once again, our values are means of all the pixels in a given trapline.

Comparison of population trends. We estimated lynx and coyote population trends in all traplines using a recently described state-space approach (Humbert et al. 2009), that provides better estimates than the predominant trend estimation method (loglinear regression of abundance data against time) which ignores the possibility of process noise. Zero counts were avoided by adding 1 to all raw counts. We first tested whether lynx population trends were skewed towards more positive values in allopatry as compared to sympatry using a one-tailed Wilcoxon rank sum test. For sympatry-only analyses, lynx population trend was the response and coyote trend the predictor. Spatial autocorrelation was accounted for by fitting a spatial simultaneous autoregressive lag model (see above). We compared the intercept-only model to the competition model (including the predictor) and selected the one with smallest AICc.

Predictive models of lynx distribution. Building on the method described by Araujo and Luoto (2007), a single measure of abundance was used for each trapline. We classified a trapline as 'lynx Common' when lynx was recorded in more than half of the years of the time series and 'lynx Rare' otherwise. The same protocol was used to determine the abundance status of coyote. We fitted Generalized Linear Models (GLM) to assess two types of relationships:

1) lynx - environment (= GLM.environment). 'Environmental' predictors were bioclimatic factors (PC1 to PC4; note that in this case, snow depth, snow cover and human influence were added to the pool of 19 climatic variables subjected to PCA to reduce the number of covariates), spatial autocorrelation (as measured for each trapline by the average value of the spatial autocovariate aut.s over the time series) and red fox abundance, as well as their quadratic term.

2) lynx - environment + coyote (= GLM.coyote). Same as 1), except that coyote abundance was added to the pool of candidate predictors.

Each GLM was calibrated using a 70\% random sample of traplines. The best model was selected using backward + forward stepwise regression and AIC criterion. Each of the two GLM was then evaluated against the remaining 30\% of the data for validation. The agreement between observed and modeled distributions was estimated using the True Skill Statistic (TSS) (Allouche et al. 2006). We also used the area under the curve (AUC) of the receiver operating characteristic (ROC)-plots as a threshold-independent measure (Guisan and Zimmermann 2000). This procedure of cross validation (i.e., calibration + validation) was repeated 20 times, yielding a distribution of 20 TSS and 20 AUC for each GLM. The accuracy (and thereby, relative merits) of each GLM was compared using Wilcoxon signed-rank tests for related samples.

Explanatory models of lynx distribution. To complement our distribution modelling approach, we developed explanatory models of lynx distribution using quantitative (instead of categorical) measures of abundance. To that end, we tested whether lynx abundance was negatively correlated with coyote abundance after controlling for a series of covariates. We developed an automatic procedure, allowing us to: i) account for repeated measures by taking residuals of a mixed model allowing a different intercept for each trapline; we did so for lynx abundance, and in a further set of control, for covariates as well, which yielded similar results (not shown); ii) perform a series of regression (linear / log-linear, whichever yielded the best fit, as estimated using R2 values) to correct lynx abundance by the value of covariates (and their quadratic terms) in the following order: bioclimatic factors (PC1 to PC4), snow depth, snow cover and human influence, spatial and temporal autocovariates, and optionally, red fox abundance. We also tested trapline area as a covariate in a subset of complementary analyses; results were unchanged (not shown); iii) test 
Table 1. Results of model selection for lynx cyclic propensity (Province-level), as measured by spectral power in 7 Canadian provinces in the center of Canada lynx's range, using basic $(\mathrm{N})$, inflation-adjusted (IA-N) or inflation-adjusted + detrended $($ IAD-N) data. Sample size $(n=38)$ corresponds to 7 provinces, with 7 time-windows for lynx except in Newfoundland where only four were available, i.e. $n=46$, of which we discarded 8 time-windows for which coyote spectral analyses could not be calculated (Ontario $=2$, Newfoundland $=2$, Quebec $=4$ ). Parameters $\mathrm{L}$ and $\mathrm{C}$ correspond to the average population sizes of lynx and coyote, respectively, during the time-window; $i=i n-$ tercepts-only model; $\mathrm{LL}=$ Log-likelihood, $\mathrm{w}=$ Akaike weights. Note that spectral power as well as lynx and coyote population sizes were obtained after time-series detrending (IAD-N data), although identical results were obtained when only spectral power was obtained after detrending (not shown); see text for further details.

\begin{tabular}{clrrrc}
\hline Data & Model & LL & AICc & $\Delta$ AICc & w \\
\hline $\mathrm{N}$ & L & -29.36 & 70.59 & 0.00 & 0.53 \\
& L + C & -28.06 & 70.83 & 0.23 & 0.47 \\
& i & -35.78 & 80.78 & 10.19 & 0.00 \\
& C & -35.75 & 83.37 & 12.78 & 0.00 \\
\hline IA-N & L & -23.09 & 58.05 & 0.00 & 0.79 \\
& L + C & -23.00 & 60.71 & 2.66 & 0.21 \\
& i & -33.22 & 75.64 & 17.59 & 0.00 \\
& C & -33.17 & 78.21 & 20.16 & 0.00 \\
\hline IAD-N & L & -25.04 & 61.95 & 0.00 & 0.80 \\
& L + C & -25.02 & 64.75 & 2.80 & 0.20 \\
& i & -33.56 & 76.33 & 14.38 & 0.00 \\
& C & -32.92 & 77.71 & 15.76 & 0.00 \\
\hline
\end{tabular}

the role of coyote on lynx abundance as measured by a simple linear regression between lynx abundance (after covariates have been accounted for) and the abundance of coyote.

All analyses were performed with R version 2.13.1 (R Development Core Team 2015).

\section{Results}

\section{I - Province-level study}

The occurrence of a gradient of lynx cyclic dynamics is illustrated by an attenuation towards the south end of the range, of: i) the average spectral power of the time-series: $\mathrm{z}$ (latitude) $=5.15, \mathrm{P}<0.001$ (Fig. A4.1; inflation-adjusted and detrended data $(\operatorname{IAD}-\mathrm{N}): \mathrm{z}=5.78)$; and ii) the proportion of time-window periods showing significant cyclic dynamics: $\mathrm{z}$ (latitude) $=13.08, \mathrm{P}<0.001$ (Fig. A4.2; IAD-N: $\mathrm{z}=7.21$ ).

In addition to evidence for a latitudinal gradient in cyclicity, we found that temporal variability of cycling propensity, as measured by the variance in spectral power, peaked at midlatitudes: $\mathrm{z}($ latitude $)=4.26, \mathrm{P}<0.001, \mathrm{z}\left(\right.$ latitude $\left.^{2}\right)=-4.11$, $\mathrm{P}<0.001$ (Fig. 1; IAD-N: $\mathrm{z}$ (latitude) $=4.69, \mathrm{z}\left(\right.$ latitude $\left.^{2}\right)=$ -4.58). Similar results were obtained using coefficient of vari- ation $(\mathrm{SD} /$ mean) of spectral power: $\mathrm{z}($ latitude $)=3.59, \mathrm{P}<$ 0.001, $\mathrm{z}\left(\right.$ latitude $\left.^{2}\right)=-3.58, \mathrm{P}<0.001(\mathrm{IAD}-\mathrm{N}: \mathrm{z}$ (latitude) $=$ 4.18, $z\left(\right.$ latitude $\left.^{2}\right)=-4.27$ : see Fig. A4.5). AICc scores for the quadratic models presented above were always $<$ AICc scores for simple linear regression and intercept-only models for the variance in spectral power ( $\triangle \mathrm{AICc}>4$, not shown). For coefficient of variation, this was also the case for IAD-N: Akaike weights for quadratic, linear regression and intercept-only were: $0.78,0.04$ and 0.18 , respectively; for our basic data $(\mathrm{N})$, however, intercept-only and quadratic models had a similar level of support $(\triangle \mathrm{AICc}=0.09$, Akaike weights $=0.47$ and 0.45 , respectively).

We tested two lines of evidence potentially supporting the influence of interspecific competition in affecting lynx cyclic propensity at mid-latitudes. Moderate support for a direct dampening effect of coyote was obtained using basic (N) data (Table 1). Although the best model only contained lynx population size as a predictor (coefficient $=0.48 \pm 0.11$ ), the model also containing coyote population size obtained non-negligible support: Akaike weight $=0.47$, coyote coefficient $=-0.17 \pm 0.10$. Ninety-five per cent Highest Posterior Density (HPD) intervals for lynx and coyote population size (' $\mathrm{L}+\mathrm{C}$ ' model in Table 1 ) were $[0.27 ; 0.76]$ and $[-0.37 ; 0.03]$, respectively, indicating a $>5 \%$ chance that the direct effect of coyote population size was actually null, or even positive. Inflation-adjusted data did not support the hypothesis of a direct dampening effect (Akaike weight $\leq 0.21$, Table 1). Finally, spectral power did not generally decrease with time, as would have been expected under a 'climatic forcing' scenario; for raw spectral power values: all $\mathrm{F}_{1,36}<0.20$, all $\mathrm{P}>$ 0.65; after other factors have been partialled out: all $\mathrm{F}_{1,5}<$ 0.11 , all $\mathrm{P}>0.76$

Three different methods were used to evaluate the potential second line of evidence for interspecific competition, that coyotes indirectly affect lynx cycles through effects on lynx population size (negative synchrony). Non-overlapping moving block permutation and overlapping moving block bootstrap approaches were consistent (Table 2). A significantly negative relationship in Saskatchewan using IA-N and timelag $=-1$ corresponded to a $95 \%$ confidence interval based on bootstrap that did not overlap zero. Marginally significant correlations were obtained at time-lag $=-1$ for Alberta (IAD-N) and Saskatchewan (all data types). Residual-based test revealed significantly negative relationships at time-lag $=-1$ for Alberta (all data types), and a similar trend, although not reaching significance, in Saskatchewan (all $\mathrm{t}<-1.07$, all $\mathrm{P}<0.15)$. Fairly consistent results were obtained using our 'order $=\max (q)$ ' procedure (Table 2$)$.

\section{II - Trapline-level study}

Comparison of population trends. Lynx population trends did not differ in allopatry and in sympatry (Table 4). In sympatry, lynx population trends were either not related to coyote trends (Alberta and Saskatchewan) or alternatively, positively rather than negatively related to coyote trends; British Columbia: $\mathrm{z}$ =7.76, $\mathrm{P}<0.001$; Ontario: $\mathrm{z}=1.46, \mathrm{P}=0.14$; Quebec: $\mathrm{z}=$ $1.87, \mathrm{P}=0.06$ (see Appendix 7 for detailed results). 
Table 2. Test of a negative synchrony between lynx and coyote time-series using basic (N), inflation-adjusted (IA-N) or inflationadjusted + detrended (IAD-N) data (Province-level). Non-overlapping block permutation and overlapping moving block bootstrap approaches yielded an estimated P-value for Spearman's rank correlation coefficient $\rho$, called $\mathrm{P}(\rho)$, and lower and upper bound of the $95 \%$ confidence interval for $\rho$, respectively. Residual-based test were conducted after pre-whitening the data in a single time-series (our ' $\operatorname{order}=\min (q)$ ' procedure) or alternatively, after removing temporal autocorrelation in both time-series, our ' $\operatorname{order}=\max (\mathrm{q})$ ' procedure. See main text and Appendix 3 for details. A significantly negative relationship was indicated by bold value, and a marginally significant $(\mathrm{P}<0.10)$ by underscores.

\begin{tabular}{|c|c|c|c|c|c|c|c|c|c|c|c|c|}
\hline \multirow[b]{3}{*}{ Province } & \multirow[b]{3}{*}{ Data } & \multirow[b]{3}{*}{ Lag } & & & & & \multicolumn{6}{|c|}{ Residual test after pre-whitening } \\
\hline & & & \multicolumn{2}{|c|}{ Block permutation } & \multicolumn{2}{|c|}{ Block bootstrap } & \multicolumn{3}{|c|}{$\operatorname{order}=\min (q)$} & \multicolumn{3}{|c|}{ order $=\max (q)$} \\
\hline & & & $\rho$ & $P(\rho)$ & $2.5 \%$ & $97.5 \%$ & $\mathrm{t}$ & $\mathrm{df}$ & $\mathrm{P}(\mathrm{t})$ & $\mathrm{t}$ & df & $\mathrm{P}(\mathrm{t})$ \\
\hline Alberta & $\mathrm{N}$ & 0 & 0.155 & 0.697 & -0.175 & 0.497 & 0.528 & 88 & 0.700 & 0.948 & 78 & 0.827 \\
\hline Alberta & $\mathrm{N}$ & -1 & -0.027 & 0.325 & -0.361 & 0.354 & -1.946 & 87 & 0.027 & -1.686 & 77 & 0.048 \\
\hline Alberta & IA-N & 0 & 0.016 & 0.395 & -0.251 & 0.356 & -0.131 & 88 & 0.448 & 0.322 & 78 & 0.626 \\
\hline Alberta & IA-N & -1 & -0.159 & 0.120 & -0.416 & 0.182 & -2.383 & 87 & 0.010 & -1.781 & 77 & 0.039 \\
\hline Alberta & IAD-N & 0 & -0.022 & 0.381 & -0.290 & 0.251 & -0.365 & 88 & 0.358 & 0.012 & 78 & 0.505 \\
\hline Alberta & IAD-N & -1 & $\underline{-0.236}$ & $\underline{0.057}$ & -0.503 & 0.030 & -2.554 & 87 & 0.006 & -2.161 & 77 & 0.017 \\
\hline Sask. & $\mathrm{N}$ & 0 & -0.118 & 0.270 & -0.410 & 0.274 & 0.013 & 88 & 0.505 & -0.384 & 82 & 0.351 \\
\hline Sask. & $\mathrm{N}$ & -1 & $-\underline{0.315}$ & $\underline{0.067}$ & -0.568 & 0.048 & -1.208 & 87 & 0.115 & -1.134 & 81 & 0.130 \\
\hline Sask. & IA-N & 0 & -0.185 & 0.168 & -0.391 & 0.193 & -0.269 & 88 & 0.394 & -0.624 & 82 & 0.267 \\
\hline Sask. & IA-N & -1 & $-\mathbf{0 . 3 5 7}$ & 0.040 & -0.535 & -0.036 & -1.073 & 87 & 0.143 & -0.559 & 81 & 0.289 \\
\hline Sask. & IAD-N & 0 & -0.169 & 0.205 & -0.424 & 0.263 & -0.300 & 88 & 0.383 & -0.644 & 82 & 0.261 \\
\hline Sask. & IAD-N & -1 & -0.341 & 0.063 & -0.561 & 0.026 & -1.090 & 87 & 0.139 & -0.561 & 81 & 0.288 \\
\hline
\end{tabular}

Table 3. Basic statistics (Trapline level). For each province, we give the number of traplines (n.trap), distribution of trapline size (m.area $=$ mean area in $\mathrm{km}^{2}(\mathrm{SD})$ ), the length of the time-series available (number of years = n.yr, starting at yr.i and ending at yr.f). We also give the mean population trend as averaged over all traplines where the species was present for lynx (l.trend) and coyote (c.trend), and the mean (SD) number of lynx (m.l) and coyote (m.c) in the whole province (trapline-1 year-1), as well as the in the lynx-coyote sympatry area (m.1.S and m.c.S, respectively).

\begin{tabular}{|c|c|c|c|c|c|c|c|c|c|c|c|}
\hline Province & n.trap & m.area & n.yr & yr.i & yr.f & 1.trend & c.trend & $\mathrm{m} .1$ & m.c & m.l.S & m.c.S \\
\hline Alberta & 1825 & $\begin{array}{r}208.3 \\
(236.6)\end{array}$ & 9 & 1998 & 2006 & -0.164 & -0.012 & $\begin{array}{r}0.33 \\
(0.86)\end{array}$ & $\begin{array}{r}2.45 \\
(6.09)\end{array}$ & $\begin{array}{r}0.56 \\
(1.09)\end{array}$ & $\begin{array}{r}4.02 \\
(7.54)\end{array}$ \\
\hline $\begin{array}{l}\text { British } \\
\text { Columbia }\end{array}$ & 2593 & $\begin{array}{r}350.9 \\
(693.8)\end{array}$ & 28 & 1982 & 2009 & -0.005 & -0.010 & $\begin{array}{r}0.29 \\
(0.92)\end{array}$ & $\begin{array}{r}0.34 \\
(1.21)\end{array}$ & $\begin{array}{r}0.80 \\
(1.42)\end{array}$ & $\begin{array}{r}0.95 \\
(1.98)\end{array}$ \\
\hline Ontario & 2726 & $\begin{array}{r}291.8 \\
(556.5)\end{array}$ & 10 & 1998 & 2007 & 0.152 & 0.032 & $\begin{array}{r}0.26 \\
(0.67)\end{array}$ & $\begin{array}{r}0.05 \\
(0.25)\end{array}$ & $\begin{array}{r}1.04 \\
(1.19)\end{array}$ & $\begin{array}{r}0.36 \\
(0.60)\end{array}$ \\
\hline Quebec & 119 & $\begin{array}{r}14240 \\
(47127)\end{array}$ & 26 & 1984 & 2009 & -0.004 & 1.317 & $\begin{array}{r}9.65 \\
(13.70)\end{array}$ & $\begin{array}{r}27.16 \\
(72.48)\end{array}$ & $\begin{array}{r}14.28 \\
(14.73)\end{array}$ & $\begin{array}{r}40.81 \\
(85.94)\end{array}$ \\
\hline Saskatchewan & 153 & $\begin{array}{r}4272.6 \\
(4169.4)\end{array}$ & 12 & 1999 & 2010 & -0.019 & 0.692 & $\begin{array}{r}0.83 \\
(0.98)\end{array}$ & $\begin{array}{r}7.17 \\
(9.54)\end{array}$ & $\begin{array}{r}1.15 \\
(0.98)\end{array}$ & $\begin{array}{r}6.77 \\
(8.16)\end{array}$ \\
\hline
\end{tabular}

Table 4. Trends allopatry vs. sympatry (Trapline level). Test of the hypothesis that lynx population trends are higher in allopatry (i.e., without coyote) than in sympatry. Correcting for prices yielded qualitatively identical results (see Appendix 6). Legend: n.A = sample size (number of traplines) in allopatry; mean. $\mathrm{A}=$ mean value of lynx population trend in allopatry; sd. $\mathrm{A}=$ standard deviation of lynx population trend in allopatry; n.S, mean.S and sd.S are sample size, mean and standard deviation for lynx population trend in sympatry; $\mathrm{W}=$ Wilcoxon rank sum test statistic, and $\mathrm{P}=\mathrm{P}$-value corresponding to a one-tailed test; see text for details.

\begin{tabular}{lrrrrrrrr}
\hline \multicolumn{1}{c}{ Province } & n.A & mean.A & sd.A & \multicolumn{1}{c}{ n.S } & mean.S & sd.S & W & P \\
\hline Alberta & 168 & -0.186 & 0.317 & 572 & -0.157 & 0.4 & 43521.5 & 0.968 \\
British Columbia & 184 & -0.006 & 0.032 & 812 & -0.005 & 0.107 & 74555 & 0.517 \\
Ontario & 667 & 0.137 & 0.293 & 212 & 0.2 & 0.334 & 59228.5 & 1.000 \\
Quebec & 5 & -0.235 & 0.214 & 79 & 0.011 & 1.228 & 128 & 0.907 \\
Saskatchewan & 6 & -0.23 & 0.235 & 102 & -0.007 & 0.24 & 145 & 0.985 \\
\hline
\end{tabular}


Table 5. Predictive models (Trapline level). The table gives the mean and standard deviation of the True Skill Statistic (TSS) for GLM. environment (e) and GLM.competition ( $\mathrm{c}=$ GLM.coyote), obtained by a 10 to 20 -fold cross validation procedure (n). Analyses were performed for all traplines (Geog = "P" for Province-wide) and for sympatric traplines only ("S"). Distributions of TSS (environment vs. competition) were compared using Wilcoxon signed-rank tests for related samples (V statistic and corresponding P-value). Using AUC instead of TSS and time-lag = -1 instead of 0 yielded similar results (see Appendix 8); some comparisons, as indicated by "” could not be performed; 'ns' means non-significant (identical distributions).

\begin{tabular}{lcrrrrrrr}
\hline \multicolumn{1}{c}{ Province } & Geog & \multicolumn{1}{c}{$\mathrm{n}$} & mean.TSS.e & sd.TSS.e & mean.TSS.c & sd.TSS.c & V.TSS & P.TSS \\
\hline Alberta & $\mathrm{P}$ & $/$ & $/$ & $/$ & $/$ & $/$ & $/$ & $/$ \\
British Columbia & $\mathrm{P}$ & 20 & 0.541 & 0.081 & 0.603 & 0.066 & 32 & 0.005 \\
Ontario & $\mathrm{P}$ & 20 & 0.649 & 0.033 & 0.646 & 0.032 & 1 & 1.000 \\
Quebec & $\mathrm{P}$ & 20 & 0.769 & 0.112 & 0.74 & 0.097 & 92 & 0.224 \\
Saskatchewan & $\mathrm{P}$ & $/$ & $/$ & $/$ & $/$ & $/$ & $/$ & $/$ \\
\hline Alberta & $\mathrm{S}$ & 20 & 0.269 & 0.126 & 0.259 & 0.111 & 33 & 0.666 \\
British Columbia & $\mathrm{S}$ & 20 & 0.337 & 0.09 & 0.433 & 0.089 & 13 & $<0.001$ \\
Ontario & $\mathrm{S}$ & 20 & 0.229 & 0.095 & 0.229 & 0.095 & 0 & $\mathrm{~ns}$ \\
Quebec & $\mathrm{S}$ & 10 & 0.543 & 0.331 & 0.582 & 0.252 & 17 & 0.944 \\
Saskatchewan & $\mathrm{S}$ & 20 & 0.469 & 0.171 & 0.46 & 0.16 & 9 & 0.787 \\
\hline
\end{tabular}

Table 6. Explanatory models (Trapline level). Results of simple linear regression (T-test and P-value) between lynx abundance (after a series of covariates have been controlled for, see main text for details) and coyote abundance. Analyses were performed for all traplines (Geog = "P" for Province-wide) and for sympatric traplines only ("S"). A total of 160 analyses were conducted (see Appendix 9) and we present here a subset corresponding to two extreme types: (1) no correction for prices, trapline or red fox abundance, and time-lag $=0$; (2) conversely, lynx abundance corrected for prices (IA-N data), and red fox abundance, time-lag $=-1$ and trapline-specific intercepts allowed.

\begin{tabular}{lcccc}
\hline \multicolumn{1}{c}{ Province } & Geog & type & \multicolumn{1}{c}{$\mathrm{t}$} & \multicolumn{1}{c}{$\mathrm{P}$} \\
\hline Alberta & $\mathrm{P}$ & 1 & 8.78 & $<0.001$ \\
Alberta & $\mathrm{P}$ & 2 & 3.33 & $<0.001$ \\
British Columbia & $\mathrm{P}$ & 1 & 50.66 & $<0.001$ \\
British Columbia & $\mathrm{P}$ & 2 & -9.37 & $<0.001$ \\
Ontario & $\mathrm{P}$ & 1 & 8.50 & $<0.001$ \\
Ontario & $\mathrm{P}$ & 2 & -1.93 & 0.053 \\
Quebec & $\mathrm{P}$ & 1 & 1.70 & 0.090 \\
Quebec & $\mathrm{P}$ & 2 & -0.91 & 0.365 \\
Saskatchewan & $\mathrm{P}$ & 1 & 5.79 & $<0.001$ \\
Saskatchewan & $\mathrm{P}$ & 2 & 0.73 & 0.465 \\
\hline Alberta & $\mathrm{S}$ & 1 & 5.96 & $<0.001$ \\
Alberta & $\mathrm{S}$ & 2 & 2.86 & 0.004 \\
British Columbia & $\mathrm{S}$ & 1 & 24.68 & $<0.001$ \\
British Columbia & $\mathrm{S}$ & 2 & -3.98 & $<0.001$ \\
Ontario & $\mathrm{S}$ & 1 & 4.60 & $<0.001$ \\
Ontario & $\mathrm{S}$ & 2 & -1.11 & 0.265 \\
Quebec & $\mathrm{S}$ & 1 & 3.97 & $<0.001$ \\
Quebec & $\mathrm{S}$ & 2 & -1.01 & 0.312 \\
Saskatchewan & $\mathrm{S}$ & 1 & 6.63 & $<0.001$ \\
Saskatchewan & $\mathrm{S}$ & 2 & 0.95 & 0.341 \\
\hline
\end{tabular}

Models of lynx distribution. For both Province-wide and sympatry-only analyses, only in British Columbia did models assuming competition (GLM.coyote) have a significantly higher predictive power than models based purely on environmental variables (GLM.environment) (Table 5; see also Appendix 8). However, the coefficient for the competitor was always positive in the 20 -fold cross validation procedure, which is contrary to the competition prediction (not shown). Consistent with this result, we found a positive relationship between lynx and coyote abundance (after covariates were controlled for) in British Columbia in 28 out of 32 run of explanatory models (Appendix 9). Overall, distribution of T-test values for the relationship between lynx and coyote abundance were skewed towards positive values (Table 6, Appendix 9), suggesting that competition with coyotes is not generally a major driver of lynx abundance.

\section{Discussion}

Interspecific competition did not seem to play a prominent role in driving lynx distribution and dynamics at the trapline level. At this smaller spatial scale, we found a general pattern of positive, rather than negative, synchrony between lynx and coyote numbers. This suggests that modest evidence supporting coyote effects on lynx detected at the province level may have been a spurious result explained by the coarser scale of the analysis, although we also emphasize the limitations of our current trapline-level study (see below). Further studies at a smaller (local) scale are warranted to get further insights into the potential impact of coyote on lynx.

\section{I-Province-level study}

The attenuation of lynx cyclic dynamics towards the south of its range (Figs. A4.1-2) apparently occurs through period lengthening, as suggested by a decrease in the period of the dominant harmonic with increasing latitude (Fig. A4.3). Ims et al. (2008) suggested that period lengthening oc- 
curs as a consequence of weakened delayed density-dependence, which can be expected if the lynx diversifies its prey selection towards the southern end of its range (see Roth et al. 2007; see also Row et al. 2014 for evidence that reduction in reproductive potential leads to drops in cyclic propensity and amplitude). Also consistent with the 'diet specialization' hypothesis, we found that variance of the period of the dominant harmonic decreases with latitude (Fig. A4.4).

In addition to some evidence for a latitudinal gradient in cyclicity, we also found that temporal variability of cycling propensity, as measured by the variance in spectral power, peaked at mid-latitudes (seven Canadian provinces from British Columbia to Newfoundland; Fig. 1). The excess variability observed at mid-latitudes occurred because transient, apparently non-cyclic periods (test for cyclicity: $\mathrm{P}$ $>0.05$ ) coexisted with regular 9-11 year cycles (Fig. 2; see also Appendix 5). Although non-cyclic periods could be due to transient loss of hare cycles at mid-latitudes, historical records show that hare cycles persisted in provinces and periods where no lynx cycles were detected (Figs. 2 and A5.2; see also Smith 1983 for source hare data).

While it may sound intuitive that lynx nearly always cycle in the north, rarely cycle in the south, and sometimes cycle in intermediate latitudes, we stress that the 'diet specialization' hypothesis does not predict the existence of temporal variability in cyclic fluctuation beyond the regular 10-year cycles themselves, only predicting fixed spatial (latitudinal) variation in lynx response to hare fluctuations (Roth et al. 2007). We thus conclude that the "diet specialization' hypothesis, while receiving support from this and other studies (e.g.,

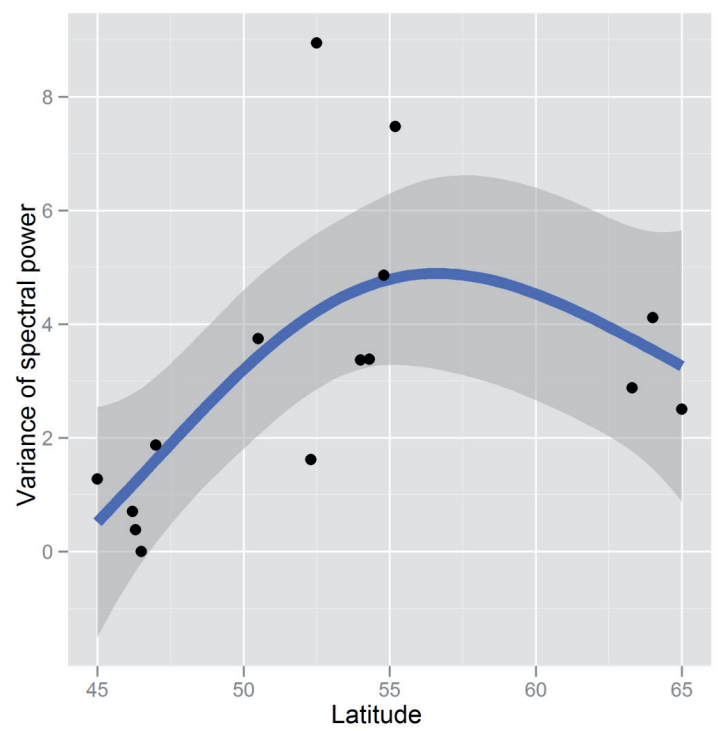

Figure 1. Absolute variability of cyclic propensity peaks at midlatitudes $(n=15)$. Of the seven 31-year time-windows, we had enough data points (i.e., $n>20$ ) to perform a spectral analysis for only one window for Washington (WA), hence it was not possible to calculate the variance for WA which was omitted from the figure. Smoothing spline (obtained by fitting a Generalized Additive Model) and $95 \%$ confidence intervals are shown for illustrative purposes (they do not take into account spatial autocorrelation in the data).
Roth et al. 2007), may not account for the whole complexity of lynx dynamics along a north-south axis.

We tested two lines of evidence potentially supporting the influence of interspecific competition in affecting lynx cyclic propensity at mid-latitudes either through a direct or indirect dampening effect of coyote. Evidence for a direct dampening effect was, at best, limited (Table 1). Conversely, the positive relationship between lynx spectral power and lynx abundance appeared to be robust (Table 1). Of course, interspecific competition with coyotes may have affected lynx spectral power 'indirectly' through coyote effects on lynx population size.

Three different methods were used to evaluate this potential second line of evidence for interspecific competition. Because there is no consensus on the 'best' method to be used, we believe that our array of methods yields a robust insight into our data (see results and Table 2 for details). We conclude that: i) competition, if any, was only detectable after considering a time-lag of one year in lynx response; ii) evidence for competition was moderate, as shown by significant P-values generally close to the threshold level of 5\%, and sensitivity of conclusions to test choice (but not so much to the type of correction applied to the data, such as detrending or correcting for pelt prices).

What may be of stronger relevance is the observation that non-cyclic periods for lynx in Alberta and Saskatchewan during the Canadian Snowshoe Rabbit Enquiry tended to correspond to statistically significant 10 -year cycles for coyote lagging one or two years behind hare peaks (Fig. 2 and A5.2). During the same period in Quebec, conversely, coyote numbers were about zero and significant lynx cycles lagging one or two years behind hare peaks were detected (Fig. 2). Results in other provinces, such as British Columbia (Fig. A5.2), also suggested a pattern whereby only the most abundant species displayed significant cyclical dynamics, apparently driven by the tracking of hare fluctuations. These results are in agreement with previous studies suggesting that coyotes can be strongly dependent on snowshoe hares, at least during cyclic highs (Todd et al. 1981, O'Donoghue et al. 1997, 1998), and further suggest that coyotes may limit the numerical tracking of hare by lynx through exploitation or interference competition. We emphasize that statistical support for this hypothesis is limited, and that further tests are necessary to robustly test its predictions.

First, it is possible that cyclic patterns in the less abundant species (such as lynx in Alberta and Saskatchewan during the Rabbit Enquiry) could not be detected because the signal-tonoise ratio was too small, independently of coyote dynamics. Our data do not allow us to discriminate between competition and this alternative. Second, due to the nature of fur harvest data, we had to merge population time-series across large spatial areas even though this could confound differences between populations occurring at smaller spatial scales. For example, the coyote may occur in prairie regions, but the lynx does not (Bayne et al. 2008). Gathering lynx and coyote data at the same spatio-temporal scales could provide a more robust test of our hypothesis, and this was the goal our traplinelevel study (see below). Third, pelt counts are subject to a 

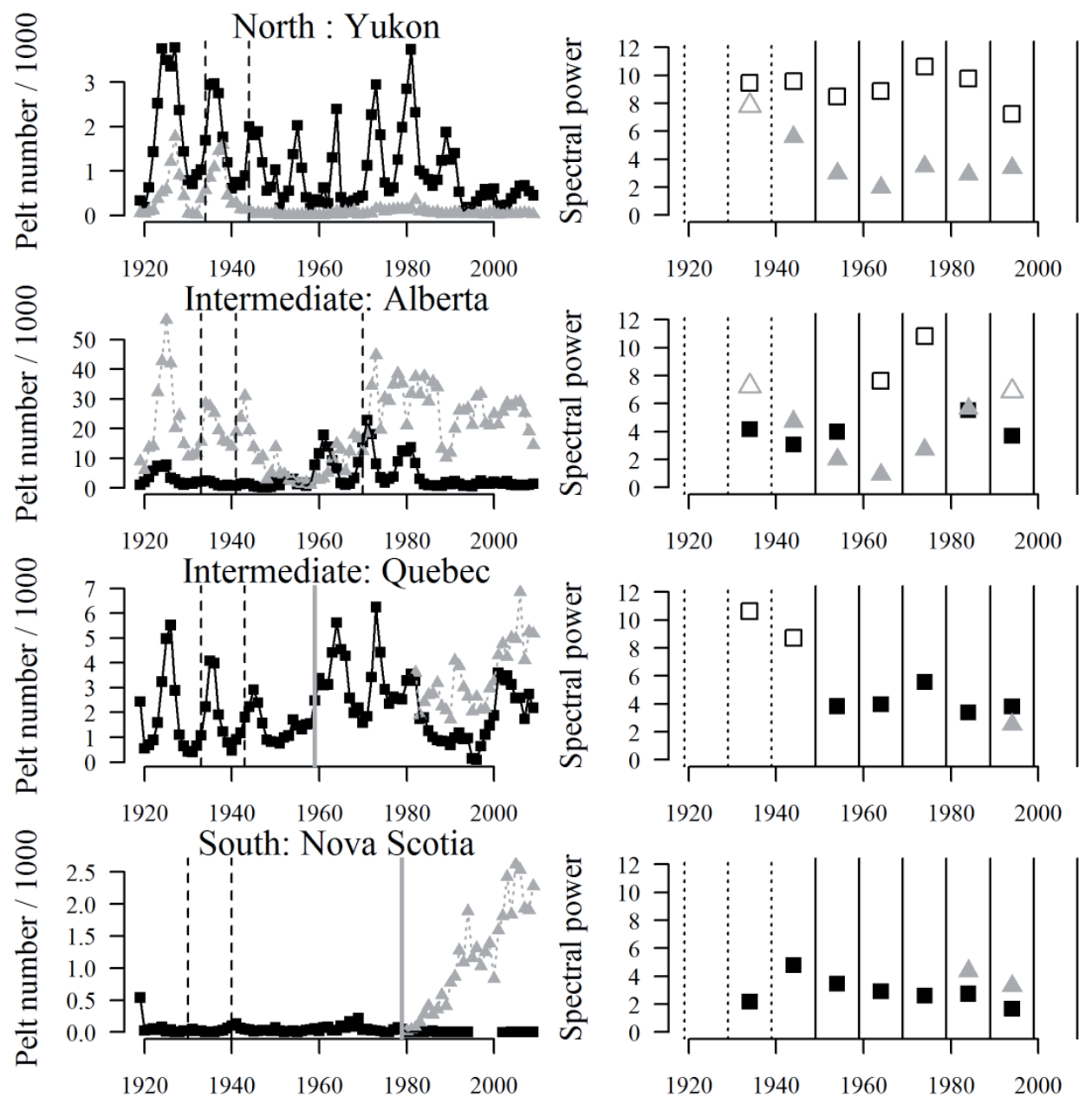

Figure 2. Latitudinal gradient of lynx cycle attenuation. Each province is representative of one out of four lynx 'cyclic dynamic' categories and occupies one row (see Appendix 5 for entire data, and discussion - 'Formulation of a hypothesis' section - for details). Left column: lynx and coyote fur harvest returns (solid black and dotted grey, respectively); solid grey vertical line, when present, represent the upper boundary of the period during which coyote started the colonization of the jurisdiction (presence pre-dating the study period when absent) based on Moore and Parker (1992) and Parker (1995); note missing coyote data in the early colonization phase in Quebec. Black vertical dashed lines represent snowshoe hare peaks during 1931-1947 (estimated after Smith 1983) plus a peak for the year 1970 in Alberta (after Brand and Keith 1979). Right column: corresponding spectral power (highest peak of the Lomb-Scargle periodogram represented on y-axis) for lynx (square) and coyote (triangle) based on a shifting window approach, after harvest data were adjusted for inflation (when data sufficient; see text for details). The 1919-2009 time-series were divided into seven windows of 31 years, starting with the period 1919-1949, and then shifting to 1929-1959, and so on. Statistical significance is indicated by open ( $\mathrm{P}<0.05$ and corresponding period: $7<\mathrm{T}$ _cycle $<13$ ), or filled $(\mathrm{P}>0.05)$ symbol.

complex set of ecological, social, and economic factors, and using fur prices as a surrogate for trapper effort can provide limited improvement over raw numbers. We found consistently positive relationships between pelt prices and various measures of harvest effort in two Canadian provinces for lynx and coyote (not shown), suggesting that pelt prices may be a suitable surrogate of harvest effort in this case, although we stress that more data may be required to validate this hypothesis. Reduced social interest in trapping may result in a mismatch between market prices and the number of licenses sold (McKelvey et al. 2010), and periodic harvest restrictions may further limit the benefit of fur price corrections (Kapfer and Potts 2012). For instance, an apparent crash in coyote harvest numbers in Alberta during the late 1940s-early 1950s (Fig.
2) coincided with withdrawal of the coyote bounty system in 1948 (Boumez 1989).

\section{II - Trapline-level study}

We found a general pattern of positive, rather than negative, synchrony between lynx and coyote harvest numbers, together with a lack of apparent ecological release in allopatry. Hence, our analyses yielded no evidence that interspecific competition with coyote plays a prominent role in driving lynx distribution and dynamics at the spatio-temporal scales investigated.

A fraction of positive synchronies detected here can result from ecological and climatic causes. In particular, there 
is evidence that lynx and coyote share a substantial fraction of their prey when they occur sympatrically (e.g., O'Donoghue 1997), but such overlap may not lead to exploitative competition if shared prey are not limiting, or if both species switch to different types of food during prey population decline. For instance, coyotes may rely extensively on hare during cyclical highs, but during periods of hare scarcity may switch to alternate prey not consumed by lynx such as livestock carrion and voles (e.g., Todd et al. 1981, O'Donoghue et al. 1998). Even lynx are capable of consuming significant amounts of alternative prey, notably red squirrel (Tamiasciurus hudsonicus (Erxleben)), when they are available (Roth et al. 2007).

We stress, however, that a lack of demonstrable effect does not necessarily imply that competition is not important. First, the relationship between the lynx and its environment was established before testing the impact of coyote. Hence, our approach followed the principle that nuisance and confounding factors should be maximized before testing a relationship of interest (Araujo and Luoto 2007, Guillaumet et al. 2008). However, this approach may underestimate the fact that the relationship with environment, such as lynx 'preference' for deep snow (Buskirk et al. 2000), may itself be a consequence of competition (Peers et al. 2013). In addition, our conclusions may not apply to the extreme southern end of the lynx range (e.g., in the United States), where we did not have any data, and where competitor densities may be highest. Finally, we could not exclude that lynx and coyote do compete but that plasticity allows them to mitigate discernible demographic responses.

The possibility that our time-series were too short to allow detection of a competition effect appears unlikely because our time-series span 9 to 28 years (Table 3), corresponding to 1-3 Canada lynx population cycles, and it is well-known that lynx can undergo drastic hare-driven population fluctuation during a single of these cycles (e.g., Krebs et al. 2001). Conversely, we acknowledge that our time-series started after the main episode of coyote expansion into eastern provinces (colonization is dated from 1900-1939 in Ontario and 1940-1959 in Quebec: see Parker 1995), and besides in Saskatchewan, our data yield little evidence of population expansion of coyote in its 'historical' western provinces (Table 3). Such a lack of definitive population increase of coyote could have dampened our ability to observe competition-related negative trends for Canada lynx.

Hence, could it be that we essentially describe in this paper an 'equilibrium', post-competition, situation? Two lines of evidence suggest that it is likely not the case. First, we note that in the province of our data set most recently colonized by coyote (Quebec), Province-wide counts (Fig. 2) are consistent with data at the trapline-level (Table 3) in showing that coyote populations were still increasing during the period covered by our time-series. Despite this, our results suggest that lynx population trends were not negatively affected by coyote population trends in Quebec; in sympatry, the slope was actually positive and marginally significant (results). Secondly, and more generally, under this 'post-competition' scenario we would still expect to find a negative impact of coyote distribution and abundance patterns on lynx distribu- tion/abundance, which was not generally the case (Tables 5-6), despite the fact that coyote were always more abundant in harvest time series than lynx (except in Ontario, Table 3).

The spatial design of sampling is also important for detecting interspecific competition. At the landscape scale, coexistence may be allowed by niche partitioning in a spatially heterogeneous environment (e.g., Amarasekare 2003). For instance, the smaller spatial requirements of red foxes apparently enabled them to persist in boundary areas adjacent to territories of coyote family groups in Maine (Harrison 1986b in Litvaitis and Harrison 1989). The spatial scale of traplines, or trapping zones that we used in the analysis, ranging from 200 (Alberta) to $>10,000 \mathrm{~km}^{2}$ (Quebec, see Table 3), may typically encompass $\geq 1$ lynx home range (Poole 2003, Vashon et al. 2008). Because a single, 'average' environmental value was used in the present study to characterize the environment at the trapping unit scale, our resolution was too coarse to test the hypothesis that lynx shifted habitat use in presence of competitors within a single trapline.

Finally, while a fraction of positive synchronies that we detected can result from common ecological and climatic causes (see above), another fraction might be explained by sampling-related bias. In particular, as noted by Weinstein (1977), trappers are 'opportunistic predators', and such generalist behavior is susceptible to generate a substantial amount of positive covariation between meso-carnivores harvests for at least two main reasons. First, many predator individuals may be captured incidentally (or not) in snares intended for others (see e.g. Lavoie et al. 2009). Secondly, it may be commonplace for trappers to trap less during years where one of their primary targets is scarce (see Novak et al. 1987), thereby yielding low returns for other species too. Conversely, a good year for the same target(s) will result in more trapping-days and artificially inflated returns in other species. In a series of 'control' analyses, we corrected lynx abundance by red fox abundance, used as a proxy of harvest effort, prior to testing the effect of coyote. These analyses were characterized by a significant reduction in positive covariation between lynx and coyote, although the distribution of T-test values (characterizing the relationship between lynx and coyote abundance) remained skewed towards positive values (not shown; but see Appendices 7 and 9). We note, however, that red fox abundance may not be the best indicator of the actual bias. The coyote is more similar to lynx in physical and ecological attributes than red fox, and this could yield a higher samplingrelated correlation in these two species' harvest.

\section{Future prospects}

Two complementary approaches may be the most promising to get further insights into the potential impact of coyote (and other putative competitors) on lynx. First, developing and contrasting habitat use models in allopatry and sympatry (or contrasting in time pre- and post-coyote era, e.g. in Quebec) may permit to determine whether lynx is subject to ecological displacement, for instance into deeper snow areas, in the presence of coyote (see Peers et al. 2013 for a case study of lynx and bobcat). Although the entire lynx's range 
may be considered, environmental values should be gathered at the site (local) scale, and not at the landscape scale as in the present study (trapline-level). Second, close up field investigations of lynx habitat use, survival, behavioral and dispersal patterns might be conducted using powerful modern tracking methods in both allopatry and sympatry with coyotes. Deeper insights might be gathered by simultaneously installing tracking devices on co-occurring coyote.

Acknowledgements: This study was funded by a Strategic Projects NSERC grant to DLM and JB, Trent University, and the Ontario Ministry of Natural Resources. We are grateful to J. Gregoire for assisting with data entry, T. McDonough for making available Alaska fur harvest statistics, R. Woolstenhulme for making available trapper effort data in Utah and Nevada, and numerous other wildlife agencies in the USA and Canada for providing data.

\section{References}

Allouche, O., A. Tsoar and R. Kadmon. 2006. Assessing the accuracy of species distribution models: prevalence, kappa and the true skill statistic (TSS). J. Appl. Ecol. 43: 1223-1232.

Alpargu, G. and J. Buonaccorsi. 2009. A model-free test for independence between time series. J. Agric. Biol. Envir. S. 14, 115-132.

Amarasekare, P. 2003. Competitive coexistence in spatially structured environments: a synthesis. Ecol. Lett. 6: 1109-1122.

Araujo, M. B. and M. Luoto. 2007. The importance of biotic interactions for modelling species distributions under climate change. Global Ecol. Biogeogr. 16: 743-753.

Bayne, E. E., S. Boutin and R. A. Moses. 2008. Ecological factors influencing the spatial pattern of Canada lynx relative to its southern range edge in Alberta, Canada. Can. J. Zool. 86: 1189-1197.

Boonstra, R. and C. J. Krebs. 2012. Population dynamics of redbacked voles (Myodes) in North America. Oecologia 168: 601-620.

Boumez, J. B. 1989. Coyote Control in Alberta. Great Plains Wildlife Damage Control Workshop. 9: 40-43.

Brommer, J. E., H. Pietiainen, K. Ahola, P. Karell, T. Karstinen and H. Kolunen. 2010. The return of the vole cycle in southern Finland refutes the generality of the loss of cycles through 'climatic forcing'. Global Change Biol. 16: 577-586.

Buonaccorsi, J. P., J. S. Elkintion, S. R. Evans and A. M. Liebhold. 2001. Measuring and testing for spatial synchrony. Ecology 82: 1668-1679.

Buskirk, S. W., L. F. Ruggiero and C. J. Krebs. 2000. Habitat fragmentation and interspecific competition: Implications for lynx conservation. In: L.F. Ruggiero et al. (eds), Ecology and Conservation of Lynx in the United States. University Press of Colorado, Boulder, Colorado. pp. 83-100.

Cattadori, I. M, D. T. Haydon, S. J. Thirgood and P. J. Hudson. 2003. Are indirect measures of abundance a useful index of population density? The case of red grouse harvesting. Oikos 100: 439-446.

Davidson, R. and J. G. MacKinnon. 2007. Bootstrap Methods In: K. Patterson and T. C. Mills (eds), Econometrics. Chapter 25 of Palgrave Handbooks of Econometrics: Vol. 1 Econometric Theory. Palgrave MacMillan, Basingstoke.

Estay, S. A., A. A. Albornoz, M. Lima, M. S. Boyce and N. C. Stenseth. 2011. A simultaneous test of synchrony causal fac- tors in muskrat and mink fur returns at different scales across Canada. PLOS ONE 6: e27766.

Fedriani, J. M., T. R. Fuller, R. M. Sauvajot and E. C. York. 2000. Competition and intraguild predation among three sympatric carnivores. Oecologia 125: 258-270.

Glynn, E. F., J. Chen and A. R. Mushegian. 2006. Detecting periodic patterns in unevenly spaced gene expression time series using Lomb-Scargle periodograms. Bioinformatics 22: 310-316.

Gosselink, T. E., T. R. Van Deelen, R. E. Warner and M. J. Joselyn. 2003. Temporal habitat partitioning and spatial use of coyotes and red foxes in East-Central Illinois. J. Wild. Manage. 67: 90103.

Guisan, A. and N. E. Zimmermann. 2000. Predictive habitat distribution models in ecology. Ecol. Model. 135: 147-186.

Guillaumet, A., J. B. Ferdy, E. Desmarais, B. Godelle and P.-A. Crochet. 2008. Testing Bergmann's rule in the presence of potentially confounding factors: a case study with three species of Galerida larks in Morocco. J. Biogeogr. 35: 579-591

Henden, J.-A., R. A. Ims, N. G. Yoccoz, P. Hellstrom and A. Angerbjorn. 2010. Strength of asymmetric competition between predators in food webs ruled by fluctuating prey: the case of foxes in tundra. Oikos 119: 27-34.

Humbert, J.-Y., S. Mills, J. S. Horne and B. Dennis. 2009. A better way to estimate population trends. Oikos 118: 1940-1946.

Ims, R.A., J.-A. Henden and S. T. Killengreen. 2008. Collapsing population cycles. Trends Ecol. Evol. 23: 79-86.

Kamler, J. F., W. B. Ballard, R. L. Gilliland, P. R. II Lemons and K. Mote. 2003. Impacts of coyotes on swift foxes in Northwestern Texas. J. Wildlife Manage. 67: 317-323

Kapfer, P. M. and K. B. Potts. 2012. Socioeconomic and ecological correlates of bobcat harvest in Minnesota. J. Wildlife Manage. 76: $237-242$

Karl, T. R., Melillo, J. M., T. C. Peterson et al. 2009. Global Climate Change Impacts in the United States. Cambridge University Press, Cambridge.

Krebs, C. J., R. Boonstra, S. Boutin and A. R. E. Sinclair. 2001. What drives the 10-year cycle of snowshoe hares. BioScience 51: 2535.

Lavoie, M., P.-Y. Collin, F. Lemieux, H. Jolicoeur, P. Canac-Marquis and S. Lariviere. 2009. Understanding fluctuations in bobcat harvest at the northern limit of their range. J. Wildlife Manage. 73: 870-875.

Levi, T. and C. C. Wilmers. 2012. Wolves-coyotes-foxes: a cascade among carnivores. Ecology 93: 921-929.

Litvaitis, J. A and D. J. Harrison. 1989. Bobcat-coyote niche relationships during a period of coyote population increase. Can. J. Zool. 67: 1180-1188.

McKelvey, S. K., E. C. Lofroth, K. P. Copeland, K. B. Aubry and A. J. Magoun. 2010. Comments on Brodie and Post: climate-driven declines in wolverine populations: causal connection or spurious correlation? Popul. Ecol. 53: 263-266.

Moore, G.C. and G.R. Parker. 1992. Colonization by the eastern coyote (Canis latrans). In: A.H. Boyer (ed.), Ecology and Management of the Eastern Coyote. Wildlife Research Unit, University of New Brunswick, Fredericton, New Brunswick, Canada. pp. p 23-37.

Murray, D. L. 2000. A geographic analysis of snowshoe hare population demography. Can. J. Zool. 78: 1207-1217.

Murray, D. L., T. D. Steury and J. D. Roth. 2008. Canada lynx research and conservation needs in the southern range: Another kick at the cat. J. Wildlife Manage. 72: 1463-1472. 
Nelson, J. L., B. L. Cypher, C. D. Bjurlin and S. Creel. 2007. Effects of habitat on competition between kit foxes and coyotes. $J$. Wildlife Manage. 71: 1467-1475.

Novak, M., M. E. Obbard, J. G. Jones, R. Newman, A. Booth, A. J. Satterthwaite and G. Linscombe. 1987. Furbearer Harvests in North America, 1600-1984. Ontario Ministry of Natural Resources, Toronto, Canada.

O’Donoghue M., S. Boutin, C. J. Krebs and E. J. Hofer. 1997. Numerical responses of coyotes and lynx to the snowshoe hare cycle. Oikos 80: 150-162.

O’Donoghue M., S. Boutin, C. J. Krebs, G. Zuleta, D. L. Murray and E. J. Hofer. 1998. Functional responses of coyotes and lynx to the snowshoe hare cycle. Ecology 79: 1193-1208.

Parker, G. R. 1995. Eastern Coyote: The Story of its Success. Nimbus Publishing, Halifax, Nova Scotia.

Peacock, E. and D. L. Garshelis. 2006. Comment on "On the Regulation of Populations of Mammals, Birds, Fish, and Insects" IV. Science 313: 45.

Peers, M. J. L., D. H. Thornton and D. L. Murray. 2013 Evidence for large-scale effects of competition: niche displacement in Canada lynx and bobcat. Proc. R. Soc. B, 280: 20132495.

Poole, K. G. 2003. A review of the Canada lynx, Lynx canadensis, in Canada. Can. Field Nat. 117: 360-376.

Ranta E., J. Lindström, H. Linden and P. Helle. 2008. How reliable are harvesting data for analyses of spatio-temporal population dynamics? Oikos, 117: 1461-1468.

R Development Core Team. 2015. R: A language and environment for statistical computing. R Foundation for Statistical Computing, Vienna, Austria. https://www.R-project.org

Ripple, W. J., A. J. Wirsing, R. L.Beschta and S. W. Buskirk. 2011. Can restoring wolves aid in lynx recovery. Wildlife Soc. B. 35: 514-518.

Ritchie E. G. and C. N. Johnson. 2009. Predator interactions, mesopredator release and biodiversity conservation. Ecol. Lett. 12: 982-998.

Roth, J. D., J. D. Marshall, D. L. Murray, D. M. Nickerson and T. D. Steury. 2007. Geographical gradients in diet affect population dynamics of Canada lynx. Ecology 88: 2736-2743.

Row, J. R., P. J. Wilson and D. L. Murray. 2014. Anatomy of a population cycle: the role of density dependence and demographic variability on numerical instability and periodicity. J. Anim Ecol. 83: $800-812$.

Scheipl, F., S. Greven and H. Kuchenhoff. 2008. Size and power of tests for a zero random effect variance or polynomial regression in additive and linear mixed models. Comput Stat. Data Anal. 52: 3283-3299.

Smith, C. H. 1983. Spatial trends in Canadian snowshoe hare, Lepus americanus, population cycles. Can. Field Nat. 97: 151-160.
Todd, A. W., L. B. Keith and C. A. Fischer. 1981. Population ecology of coyotes during a fluctuation of snowshoe hares. J. Wildlife Manage. 45: 629-640.

US Fish and Wildlife 2000. 50 CFR Part 17 - Endangered and Threatened Wildlife and Plants; Determination of Threatened Status for the Contiguous U.S. Distinct Population Segment of the Canada Lynx and Related Rule; Final Rule. Available at http://www.fs.usda.gov/Internet/FSE_DOCUMENTS/ stelprdb5193136.pdf

Vashon, J. H., A. L. Meehan, W. J. Jakubas, J. F. Organ, A. D. Vashon, C. R. McLaughlin, G. J. Matula, Jr. and S. M. Crowley. 2008. Spatial ecology of a Canada lynx population in northern Maine. J. Wildlife Manage. 72: 1479-1487.

Weinstein, M. S. 1977. The hares, lynx, and trappers. Am. Nat. 111: 806-808.

Zhang, X., Brown, R., Vincent, L., Skinner, W., Feng, Y. and E. Mekis. 2011. Canadian Climate Trends, 1950-2007. Canadian Biodiversity: Ecosystem Status and Trends 2010, Technical Thematic Report No. 5. Canadian Councils of Resource Ministers. Ottawa, ON

Received June 14, 2014

Revised May 10, 2015

Accepted June 13, 2015

\section{Electronic supplements}

Appendix 1. Harvest data sources for Province-level study

Appendix 2. Inflation-adjusted pelt harvests.

Appendix 3. Methods to assess the synchrony between lynx and coyote time-series.

Appendix 4. Additional figures for Canada lynx dynamics (Province-level study).

Appendix 5. Complement to Fig. 2, wherein are shown all jurisdictions.

Appendix 6. Complement to Table 4 (Trends allopatry vs. sympatry), wherein all performed analyses are presented.

Appendix 7. Relationship between lynx population trends and coyote population trends in sympatry.

Appendix 8. Complement to Table 5 (Predictive models), wherein all performed analyses are presented.

Appendix 9. Complement to Table 6 (Explanatory models), wherein all performed analyses are presented.

The file may be downloaded from www.akademiai.com. 